\title{
LncRNA NEAT1 is involved in temozolomide resistance by regu- lating MGMT in glioblastoma multiforme
}

\author{
Kangli $\mathrm{Xu}^{\mathrm{a}}$, Qingsheng $\mathrm{Xu}^{\mathrm{a}}$, Zhanxiong $\mathrm{Wu}^{\mathrm{b}}$, Shengjie $\mathrm{Xu}^{\mathrm{c}}$, Jian Shen ${ }^{\mathrm{a} *}$ \\ ${ }^{a}$ Department of Neurosurgery, The First Affiliated Hospital, School of Medicine, Zhejiang University, Hangzhou, China \\ ${ }^{b}$ Department of Cardiology, Sir Run Run Shaw Hospital, School of Medicine, Zhejiang University, Hangzhou, China \\ 'School of Electronic Information, angzhou Dianzi University
}

\begin{abstract}
Introduction: As one of the most aggressive and lethal tumors, glioblastoma multiforme (GBM) is commonly treated by surgical resection combined with radiotherapy and chemotherapy. Temozolomide (TMZ) is the preferred chemotherapy medicine against GBM. However, recurrent GBM patients exhibit TMZ resistance. As reported in previous studies, NEAT1 is over-expressed in glioma cells; thus, we explored the relationship between NEAT1 and TMZ resistance in GBM.

Materials and Methods: The expression levels of NEAT1 and 0-6-methylguanine-DNA methyltransferase (MGMT) in GBM tissues and cells were determined by quantitative real-time PCR. Si-RNA and the overexpression vector were transfected into GBM cells to modulate the level of related molecules. Western blot analysis was used to investigate the protein expression of MGMT. The cell viability and IC-50 were analyzed by CCK-8, and apoptosis was detected by flow cytometry assay.

Result: The expression level of NEAT1 in the TMZ-sensitive GBM tissues and cells was lower than that in the TMZ-resistant GBM tissues and cells. Furthermore, due to the down-regulation of NEAT1 in TMZ-resistant GBM cells transfected with Si-RNA, the viability and IC-50 value of the GBM cell lines were decreased, and the knockdown of NEAT1 significantly enhanced TMZ-induced cell apoptosis in GBM cells. We also determined that the mRNA and protein level of MGMT were up-regulated in TMZ-resistant GBM cells. Interference with MGMT expression led to a decrease in viability and IC-50 value in the GBM cells, and the knockdown of NEAT1 suppressed the transcription and translation levels of MGMT. However, the over-expression of MGMT enhanced TMZ resistance in NEAT1-silenced U87 and U251 cells.

Conclusion: NEAT1 participates in the TMZ resistance of GBM cells by regulating MGMT.

Keywords: IncRNA NEAT1, glioblastoma multiforme, Temozolomide, 0-6-methylguanine-DNA methyltransferase
\end{abstract}

\section{INTRODUCTION}

Among all central nervous system cancers, glioma is the primary tumor and the most common ${ }^{[1,2]}$. Glioblastoma multiforme (GBM) is one of the most malignant types of glioma, with a poor patient survival rate ${ }^{[3]}$. After a confirmed diagnosis, the therapy for GBM includes radiotherapy, chemotherapy, and surgical resection. Temozolomide (TMZ), which damages DNA strands via the methylation of the 0-6-methylguanineDNA methyltransferase (MGMT) promoter, is the most effective chemotherapy in clinical application for providing hope to GBM patients ${ }^{[4]}$. However, in longterm therapy, some GBM patients exhibit hardly any sensitivity to TMZ, and recrudescent GBM patients

Corresponding author: Jian Shen, M.D.

Mailing address: Department of Neurosurgery, The First Affiliated Hospital, School of Medicine, Zhejiang University, Address: 79 Qingchun Rd., Hangzhou 310003, People's Republic of China.

E-mail: 1314006@zju.edu.cn

Received: 20 January 2018 Accepted: 20 March 2018 also develop TMZ resistance, suppressing the efficacy of TMZ chemotherapy ${ }^{[5]}$. It has been reported that MGMT is over-expressed in glioblastomas, enhancing resistance to $\mathrm{TMZ}^{[6]}$. Nevertheless, several studies indicate that the up-regulation of MGMT cannot completely prevent TMZ resistance in GBM ${ }^{[7,8]}$. Hence, it is important to explore the underlying mechanisms of chemoresistance to TMZ.

Long non-coding RNA (lncRNA), a single-strand RNA with about 200 nucleotides, has been found to be associated with many physiological and pathological cell processes, such as embryonic development, cell differentiation, proliferation, and tumorigenesis ${ }^{[9]}$. On the other hand, IncRNAs perform the duties of molecular mediators, including cellular signaling, acting as molecular decoys, and contributing to scaffold modeling ${ }^{[10]}$. Therefore, lncRNAs play a pivotal role in the growth of tumors. Without exception, lncRNA nuclear-enriched abundant transcript 1 (NEAT1) has been defined as an oncogene in many human cancers, altering the epigenetic landscape of target gene 
promoters to drive cell growth in prostate cancer ${ }^{[11]}$, contributing to the poor survival rate of breast cancer patients ${ }^{[12]}$, and advancing the progression of nonsmall-cell lung cancer ${ }^{[13]}$. As reported in previous studies, NEAT1 is over-expressed in glioma cells, promoting glioma tumorigenesis and impacting the glioma prognosis ${ }^{[14,15]}$. However, the influence of IncRNA NEAT1 on TMZ resistance in GBM has been unclear.

In this study, we first defined the expression level of NEAT1 in TMZ-resistant GBM cells and the impact of the down-regulation of NEAT1 on the TMZ resistance of these cells. In addition, we explored the potential relationship between NEAT1 and MGMT in the TMZ resistance of these cells. Our study offers a novel angle on the remission of TMZ resistance in GBM.

\section{MATERIALS AND METHODS}

Patients and specimens

There were 112 GBM patients enrolled in our study. Of these, 40 were diagnosed with initial glioblastoma multiforme, had not undergone chemotherapy or radiotherapy, and were sensitive for TMZ. The glioblastoma multiforme of the other 72 patients, who were resistant to TMZ, was recrudescent. All patients were treated with surgical resection at our hospital. The tissues obtained were stored with liquid nitrogen after the written approval of the Institutional Research Ethics Board and the informed consent of all enrolled patients. This study was supported by Ethics committee of The First Affiliated Hospital, Zhejiang University. The expression of NEAT1 in these GBM tissues was determined by quantitative reverse transcription real-time PCR.

\section{Cell lines}

Human glioblastoma multiforme cell lines U87 and U251, which were purchased from the Cell Culture Center of the Chinese Academy of Medical Sciences (Beijing, China), were cultured in DEME medium containing 10\% FBS, $100 \mathrm{U} / \mathrm{mL}$ of penicillin, and 100 $\mu \mathrm{M}$ of streptomycin at $37^{\circ} \mathrm{C}$ in a humid environment with $5 \% \mathrm{CO} 2$. The method of obtaining the TMZresistant cell lines U87-R and U251-R has been described previously ${ }^{[16]}$. Briefly, after being maintained with $10 \mu \mathrm{M}$ of TMZ for two weeks, the GBM cell lines U87 and U251 were sequentially exposed to TMZ, the concentration of which was enhanced about twofold every two stages until it reached the maximum concentration, which could lead to the death of all resistant cells. The TMZ-resistant cell lines U87-R and U251-R were induced to be established at $200 \mu \mathrm{M}$ of TMZ.

\section{Cell transfection}

Si-NEAT1, used to suppress NEAT1 expression; SiMGMT, used to depress MGMT expression; and MGMT-pcDNA vector, used to over-express MGMT, were purchased from Ribobio (Guangzhou, China). The non-targeting control Si-RNA (Mock) and the empty vector (pcDNA) served as negative controls. After being cultured for 24 hours, the GBM cell lines were performed for transfection by Opti-MEM I and Lipofectamine 3000 (Invitrogen, CA, USA) using the standard system of the manufacturer's protocol. To obtain stable cell lines, G418 (Invitrogen, CA, USA) was added to the selection medium.

\section{RNA isolation and qRT-PCR}

TRIzol reagent (Invitrogen, San Diego, CA, USA) was used to extract the total RNA following the manufacturer's protocol. After the extracted RNA was reversely transcribed to cDNA, the resultant was used with the miRNA qPCR Quantitation Kit (Genepharma, Shanghai, China) to perform quantitative real-time PCR in the ABI PRISM 7300 RT-PCR system (Applied Biosystems, Foster, CA, USA). The $2^{-\Delta \Delta \mathrm{Ct}}$ method was used to carry the related level of RNA expression.

\section{Cell viability with TMZ and IC-50 value}

The GBM cells were seeded in 96-well plates with $100 \mu \mathrm{M}$ of TMZ and cultured for $24 \mathrm{~h}, 48 \mathrm{~h}, 72 \mathrm{~h}$, and 96h. At the time of harvest, $10 \mu \mathrm{L}$ of CCK-8 (Roche Biochemicals, Mannheim, Germany) were added to every well. After 4 hours, the cell viability was indicated by the absorbance value, measured at $450 \mathrm{~nm}$. IC-50, the half-maximal inhibitory concentration, represented the concentration of TMZ required for $50 \%$ inhibition of the GBM cells. The IC-50 value was detected by the CCK-8 reagent and calculated by the Graph Pad Prism software (Graph Pad Software, La Jolla, CA, USA).

\section{Cell apoptosis}

The GBM cells were stained using a V-FITC/PI double staining kit (Key GEN, Shanghai, China) according to the manufacturer's protocol. The apoptosis of the GBM cells was surveyed by flow cytometry (FACScan, BD Biosciences).

\section{Western blotting assay}

The western blotting assay process has been described previously ${ }^{[17]}$. The antibodies against MGMT and GADPH, regarded as loading controls, were purchased from NeoMarkers (Fremont, CA, USA). The protein bands were imaged by a Fluor S Multi-Imager (Bio-Rad Laboratories, Hercules, CA, USA).

Statistical analysis 


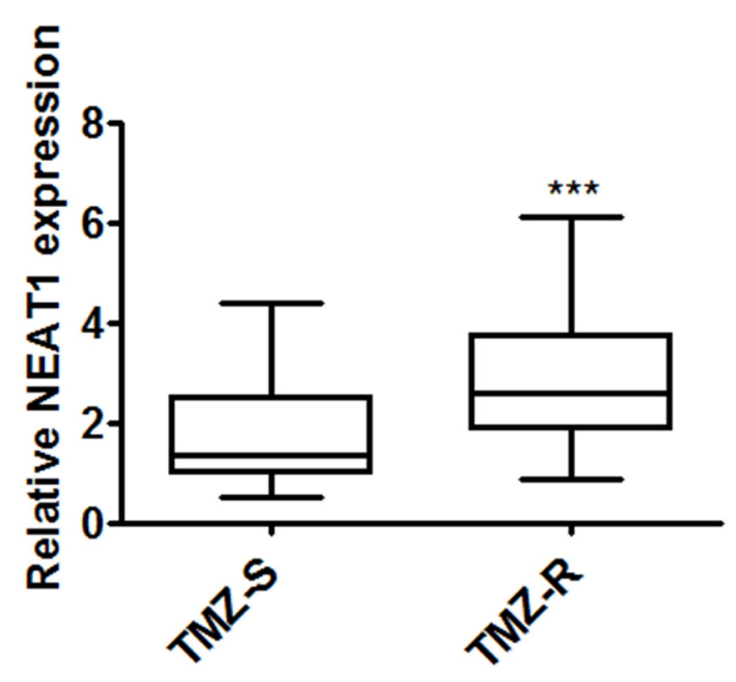

Figure 1 The expression of NEAT1 in GBM tissues. The expression of NEAT1 in TMZ-resistant GBM tissues was higher than that in TMZ-sensitive GBM tissues $\left({ }^{* * *} \mathrm{P}<0.001\right)$.

All data were analyzed using the SPSS 20.0 software (SPSS, Chicago, IL, USA) and the Graph Pad Prism software (Graph Pad Software, La Jolla, CA, USA) and expressed as mean $\pm \mathrm{SD}$. Student's test, the chi-square test, or one-way ANOVA were used to calculate the significance of the differences. A P value of less than 0.01 was considered statistically significant.

\section{RESUITS}

NEAT1 was over-expressed in the recrudescent GBM tissues with TMZ resistance.

For analyzing the effect of NEAT1 expression on TMZ resistance, 40 patients who were initially diagnosed with GBM and detected as sensitive to TMZ and 74 recrudescent GBM cases with TMZ resistance were gathered. The expression of NEAT1 in the tumor tissues of the two groups was defined, and NEAT1 expression was evidently up-regulated in the recrudescent patients (Figure 1).

NEAT1 over-expression was associated with TMZ resistance in the GBM cells.

As the data above show a higher level of NEAT1 expression in the TMZ-resistant cases, the TMZresistant cell lines U87-R and U251-R, which were continuously exposed in the senior concentration of TMZ, were obtained. According to the CCK-8 assays, the TMZ-resistant cell lines U87-R and U251-R indicated obvious growth superiority with $100 \mu \mathrm{M}$ of TMZ (Figure 2A). Because of their enhanced resistance, the U87-R and U251-R cells revealed the higher IC-50 values ( $\geq$ three fold) compared to the U87 and U251 cells (Figure 2B). Similarly, the expression of NEAT1 was enhanced (>four fold) in the U87-R and U251-R cells (Figure 2C).

The knockdown of NEAT1 inhibited TMZ resistance and promoted cell apoptosis in the GBM cells.

To further explore the role of NEAT1 in GBM cells, the U87-R and U251-R cells were transfected with Si-RNA, which interfered with NEAT1 regulation. As shown in Figure $3 \mathrm{~A}$, compared with those in the controls, the levels of NEAT1 expression were markedly lower in the U87-R-Si-NEAT1 and U251-R-Si-NEAT1 cells. In accordance with the CCK-8 assays, the cell viability with $100 \mu \mathrm{M}$ of TMZ and the IC-50 value of the U87-R and U251-R cells transfected with Si-NEAT1 were visibly reduced compared to those of the control cells (Figure 3B and 3C). Meanwhile, the down-regulation of NEAT1 resulted in a remarkable increase in apoptotic cells in the U87-R and U215-R cells cultured with $100 \mu \mathrm{M}$ of TMZ (Figure 3D and 3E).

The down-regulation of NEAT1 depressed the expression of MGMT.

Because a large number of studies have reported that MGMT is related to the TMZ resistance of GBM cells, qRT-PCR and western blot assays were performed. The
A

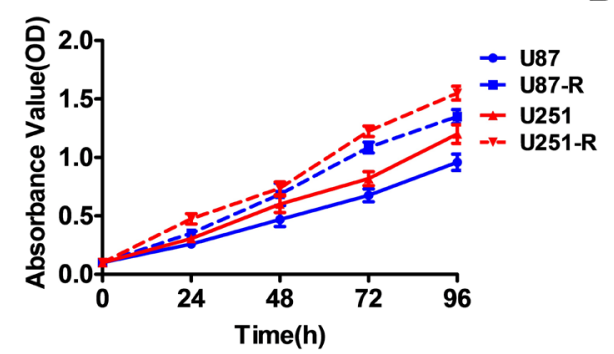

B

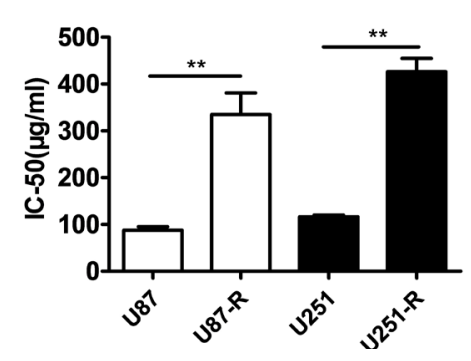

C

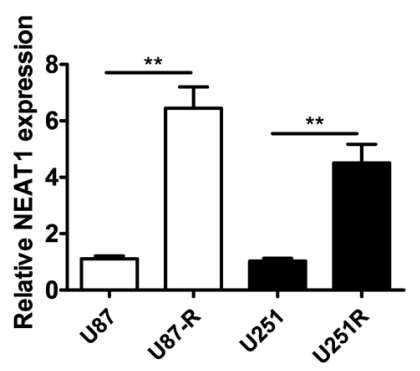

Figure 2 NEAT1 is up-expressed in TMZ-resistant GBM cell lines. (A) The viability of GBM cells was detected using CCK-8 assays after exposure to TMZ for $24 \mathrm{~h}, 48 \mathrm{~h}, 72 \mathrm{~h}$, and $96 \mathrm{~h}$. The absorbance values were measured at $450 \mathrm{~nm}$. (B) The IC-50 of TMZ in the GBM cells was assessed by CCK-8 assays ( $\left.{ }^{* *} \mathrm{P}<0.01\right)$. (C) The expression of NEAT1 in TMZ-resistant GBM cell lines was found to be up-regulated via qRTPCR analysis $(* * \mathrm{P}<0.01)$. 
A

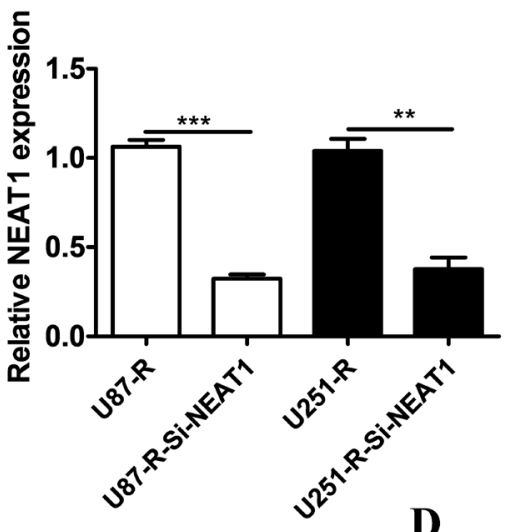

B

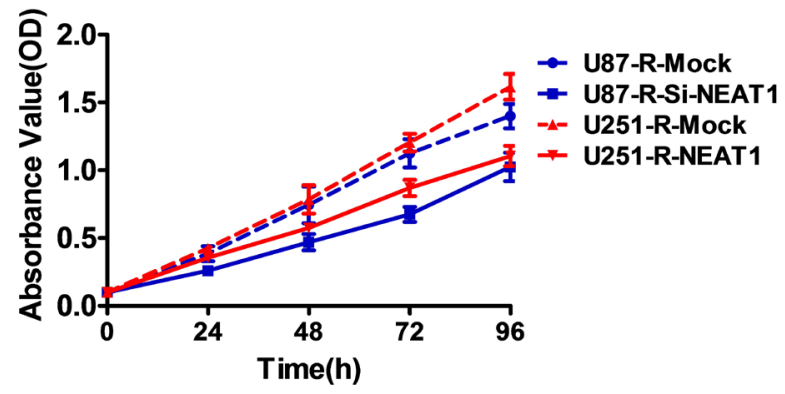

C

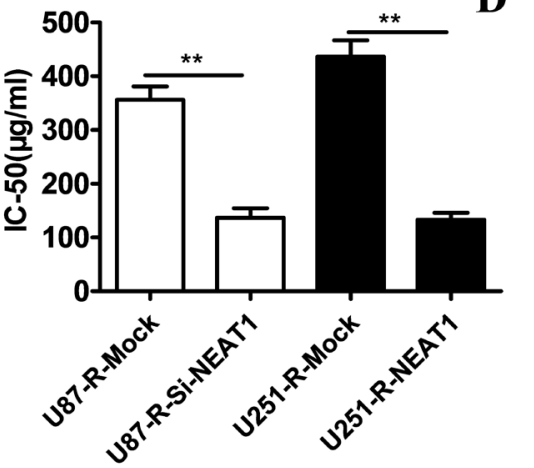

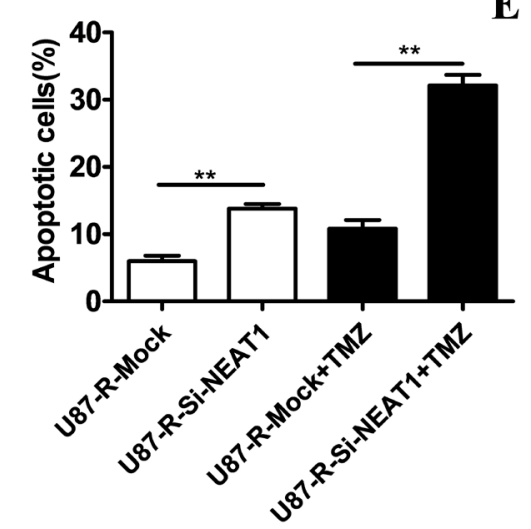

$\mathbf{E}$

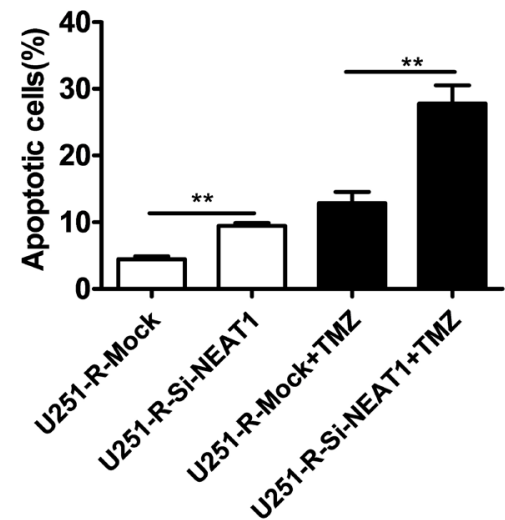

Figure 3 Knockdown of NEAT1 promotes TMZ sensitivity and cell apoptosis. (A) The expression of NEAT1 in TMZ-resistant GBM cell lines was defined using qRT-PCR analysis $\left({ }^{* *} \mathrm{P}<0.01,{ }^{* * *} \mathrm{P}<0.001\right)$. (B) The cell viability of TMZ-resistant GBM cells was detected using CCK8 assays after exposure to TMZ for $24 \mathrm{~h}, 48 \mathrm{~h}, 72 \mathrm{~h}$, and $96 \mathrm{~h}$. The absorbance values were measured at $450 \mathrm{~nm}$. (C) The IC-50 of TMZ in the TMZ-resistant GBM cells was assessed by CCK- 8 assays $\left({ }^{* *}<<0.01\right)$. (D) The influence of NEAT1 down-regulation on the apoptosis of U87-R cells cultured with TMZ was determined by flow cytometry analysis $\left({ }^{* *} \mathrm{P}<0.01\right)$. (E) The influence of NEAT1 down-regulation on the apoptosis of U251-R cells cultured with TMZ was determined by flow cytometry analysis $(* * \mathrm{P}<0.01)$.

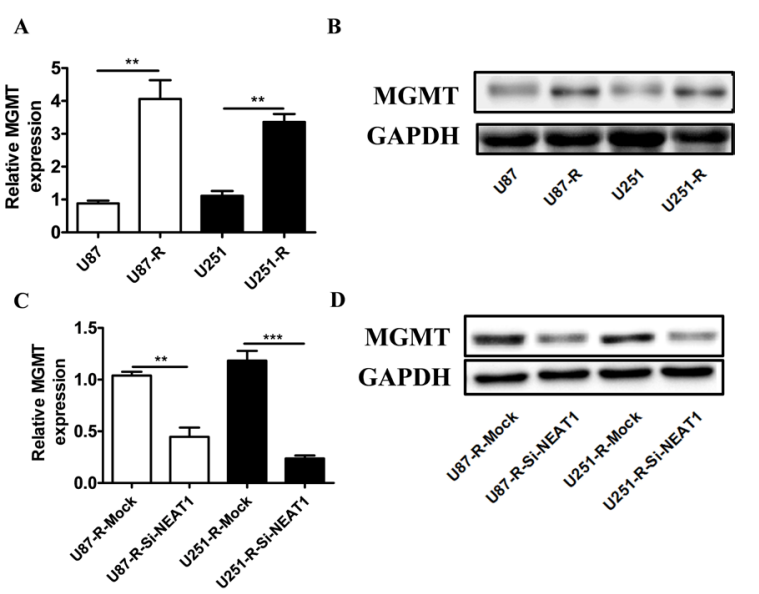

Figure 4 Knockdown of NEAT1 depressed the expression of MGMT. (A) The mRNA expression of MGMT in TMZ-resistant GBM cell lines was found to be up-regulated via qRT-PCR analysis $(* * P<0.01)$. (B) The protein expression of MGMT in TMZ-resistant GBM cell lines was determined to be up-regulated via western blot analysis. (C) The expression level of MGMT mRNA in TMZ-resistant GBM cell lines was defined using qRTPCR analysis ( $\left.{ }^{* *} \mathrm{P}<0.01,{ }^{* * *} \mathrm{P}<0.001\right)$. (D) The protein level of MGMT expression in TMZ-resistant GBM cell lines was detected using western blot analysis. results showed that the mRNA and protein expression levels of MGMT in the U87-R and U215-R cells were significantly enhanced compared to those in the U87 and U251 cells (Figure 4A and 4B), while the levels of MGMT mRNA and protein expression in the U87-RSi-NEAT1 and U251-R-Si-NEAT1 cells were evidently suppressed by NEAT1 deletion (Figure 4C and 4D).

\section{NEAT1 knockdown and the over-expression of MGMT impacted TMZ resistance in the GBM cells.}

The CCK-8 assays illustrated that because of the downregulation of MGMT in the U87-R and U251-R cells, the IC-50 value and cell viability with $100 \mu \mathrm{M}$ of TMZ in the U87-R-Si-MGMT and U251-R-Si-MGMT cells were inhibited compared to those of the controls (Figure $5 \mathrm{~A}$ and $5 \mathrm{~B}$ ). Also, the IC-50 and cell viability with 100 $\mu \mathrm{M}$ of TMZ in the U87-R-Si-NEAT1+MGMT and U251R-Si-NEAT1+MGMT cells were obviously increased with the over-expression of MGMT (Figure 5C and 5D). In addition, the down-regulation of NEAT 1 combined with TMZ could significantly advance the apoptosis of the U87-R and U251-R cells, while the up-regulation of MGMT in the U87-R-Si-NEAT1+MGMT and U251-R- 


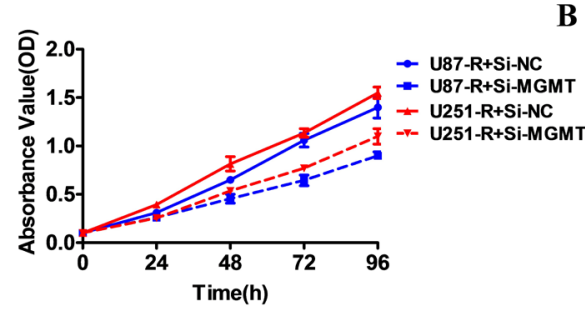

C

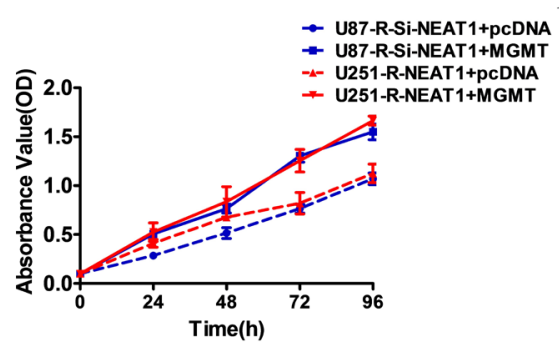

E

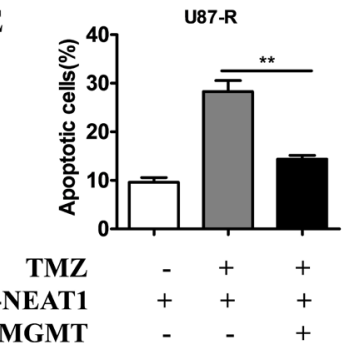

B

D
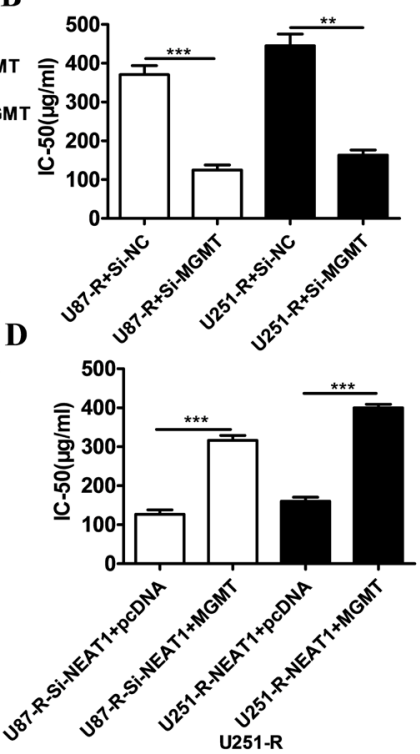

F

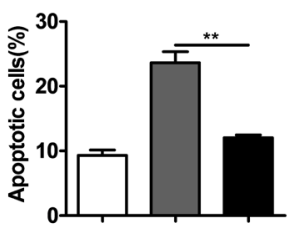

TMZ

Si-NEAT1 MGMT

\section{DOI: $10.31491 /$ CSRC.2018.3.011}

Figure 5 The expression of NEAT1 and MGMT impacted TMZ resistance in GBM cells. (A) The cell viability of TMZ-resistant GBM cells, which were transfected with Si-MGMT, was detected using CCK- 8 assays after exposure to TMZ for $24 \mathrm{~h}, 48 \mathrm{~h}, 72 \mathrm{~h}$, and $96 \mathrm{~h}$. The absorbance values were measured at $450 \mathrm{~nm}$. (B) The IC-50 of TMZ in the TMZ-resistant GBM cells that were transfected with Si-MGMT was assessed by CCK-8 assays $\left({ }^{* *} \mathrm{P}<0.01\right)$. (C) The cell viability of the TMZ-resistant GBM cells that were transfected with Si-NEAT1 and MGMT-pcDNA was detected using CCK-8 assays after exposure to TMZ for $24 \mathrm{~h}, 48 \mathrm{~h}, 72 \mathrm{~h}$, and $96 \mathrm{~h}$. The absorbance values were measured at $450 \mathrm{~nm}$. (D) The IC-50 of TMZ in the TMZ-resistant GBM cells that were transfected with $\mathrm{Si}-$ NEAT1 and MGMT-pcDNA was assessed by CCK- 8 assays $\left({ }^{* *} \mathrm{P}<0.01\right)$. (E) The influence of TMZ and MGMT up-regulation on the apoptosis of U87-R cells transfected with Si-NEAT1 was evaluated by flow cytometry analysis $(* * \mathrm{P}<0.01)$. (F) The influence of TMZ and MGMT up-regulation on the apoptosis of U251-R cells transfected with Si-NEAT1 was investigated by flow cytometry analysis $(* * \mathrm{P}<0.01)$.
Si-NEAT1+MGMT cells combined with TMZ lowered cell apoptosis in comparison with that of the U87-R-SiNEAT1 and U251-R-Si-NEAT1 cells with TMZ (Figure $5 \mathrm{E}$ and $5 \mathrm{~F}$ ).

\section{DISCUSSION}

As one of the most aggressive and lethal tumors, GBM is commonly treated by surgical resection combined with radiotherapy and chemotherapy ${ }^{[18,19]}$. TMZ is the preferred chemotherapy medicine against GBM, for which there are very few efficient drug treatments ${ }^{[20]}$. Another unhopeful situation is that recurrent GBM patients exhibit TMZ resistance following first-time chemo-treatments with $\mathrm{TMZ}^{[21]}$. Many studies have found that lncRNAs are involved in various biological processes in cancer cells, such as cell cycle control, transcriptional and translational regulation, apoptosis, cell invasion, and cell migration ${ }^{[22-25]}$.

Large amounts of evidence reveal that the chemoresistance of tumors is associated with lncRNAs. For instance, the lncRNA HOTTIP contributes to gemcitabine resistance in pancreatic cancer ${ }^{[26]}$, the lncRNA UCA1 enhances cisplatin/gemcitabine resistance in bladder cancer cells ${ }^{[27]}$, and the lncRNA
PVT1 promotes multidrug resistance in gastric cancer ${ }^{[28]}$. Although it has been reported that the lncRNA NEAT1 is over-expressed in GBM tissues compared with healthy brain tissues ${ }^{[14]}$, the function of NEAT1 in TMZ resistance has not been previously studied. Comparing lncRNA NEAT1 between recrudescent GBM tissues and initial GBM tissues, we found that NEAT1 expression was increased in the recrudescent tissues. Meanwhile, the expression level of NEAT1 in TMZsensitive cells (U87 and U251) was lower than that in TMZ-resistant cells (U87-R and U251-R), suggesting that NEAT1 is associated with TMZ resistance in GBM cells. The different expression levels of NEAT1 in GBM provide potential prognosis values.

Furthermore, the up-regulation or down-regulation of lncRNAs could change the progression of tumors. The knockdown of TUG1 by SiRNA has obviously depressed cell proliferation and promoted cell apoptosis in colon cancer cells ${ }^{[29]}$, while the over-expression of the lncRNA GAS5 may inhibit cell growth and enhance gefitinib sensitivity in lung adenocarcinoma cells ${ }^{[30]}$. The knockdown of CARLo-5 in gastric cancer cell lines has markedly suppressed cell proliferation by inducing G0/G1 cell-cycle arrest and apoptosis ${ }^{[31]}$. Similarly, due 
to the down-regulation of NEAT1 in TMZ-resistant GBM cells transfected with Si-RNA, the viability and IC-50 value of the GBM cell lines were decreased. This result shows that interfering with NEAT1 expression has the benefit of depressing TMZ resistance in GBM cells. Further, the knockdown of NEAT1 significantly enhanced TMZ-induced cell apoptosis in GBM cells. In other words, the TMZ sensitivity of GBM cells could be significantly improved. This result suggests a novel target for enhancing the efficacy of TMZ chemotherapy. Previous studies have shown that the dysregulation of MGMT is the primary cause of TMZ resistance in GBM cells ${ }^{[17,32,33]}$. Similarly, we determined that the mRNA and protein levels of MGMT were up-regulated in TMZ-resistant GBM cells. Interfering with MGMT expression led to decreases in the viability and IC-50 value of the GBM cells, equal to those resulting from NEAT1 silencing. Moreover, the knockdown of NEAT1 suppressed the transcription and translation levels of MGMT. However, the over-expression of MGMT in NEAT1-silenced GBM U87 and U251 cells enhanced these cells' TMZ resistance.

In summary, not only was the expression of NEAT1 enhanced in TMZ-resistant GBM cells, but NEAT1 was involved in the TMZ resistance of GBM cells by regulating MGMT.

\section{ACKNOWLEDGEMENTS}

This work was supported by grants of Zhejiang Provincial Natural Science Foundation (no. LY16H090004), National Natural Science Foundation of China (no. 81501065), Zhejiang Provincial Traditional Chinese Medicine Science and Technology Plan (no.2016ZA115). Zhejiang Provincial Medical and Health Science and Technology Plan (no.2015KYA091).

\section{REFERENCES}

1. Siegel, R., Ma, J., Zou, Z., and Jemal, A. (2014) Cancer statistics, 2014. CA: A Cancer Journal for Clinicians 64, 9-29

2. Jemal, A., Bray, F., Center, M. M., Ferlay, J., Ward, E., and Forman, D. (2011) Global cancer statistics. CA: A Cancer Journal for Clinicians 61, 33-64, 31

3. Johnson, D. R., and O'Neill, B. P. (2012) Glioblastoma survival in the United States before and during the temozolomide era. Journal of Neuro-Oncology 107, 359-364

4. Jiang, G., Li, L. T., Xin, Y., Zhang, L., Liu, Y. Q., and Zheng, J. N. (2012) Strategies to improve the killing of tumors using temozolomide: targeting the DNA repair protein MGMT. Current Medicinal Chemistry 19, 3886-3892

5. Caldera, V., Mellai, M., Annovazzi, L., Monzeglio,
O., Piazzi, A., and Schiffer, D. (2012) MGMT hypermethylation and MDR system in glioblastoma cancer stem cells. Cancer Genomics \& Proteomics 9, 171-178

6. Taylor, J. W., and Schiff, D. (2015) Treatment Considerations for MGMT-Unmethylated Glioblastoma. Current Neurology and Neuroscience Reports 15, 507-507

7. Z, Y., G, Z., G, X., L, Z., Y, C., H, Y., Z, Z., C, L., and Y, L. (2015) Metformin and temozolomide act synergistically to inhibit growth of glioma cells and glioma stem cells in vitro and in vivo. Oncotarget 6, 32930-32943

8. Zhang, J., Stevens, M. F., and Bradshaw, T. D. (2012) Temozolomide: mechanisms of action, repair and resistance. Current Molecular Pharmacology 5, 102-114

9. Wilusz, J. E. (2015) Long noncoding RNAs: Rewriting dogmas of RNA processing and stability. Biochimica et biophysica acta 1859, 128-138

10. Zhang, X., Sun, S., Pu, J. K., Tsang, A. C., Lee, D., Man, V. O., Lui, W. M., Wong, S. T., and Leung, G. K. (2012) Long non-coding RNA expression profiles predict clinical phenotypes in glioma. Neurobiology of Disease 48, 1-8

11. Chakravarty, D., Sboner, A., Nair, S. S., Giannopoulou, E., Li, R., Hennig, S., Mosquera, J. M., Pauwels, J., Park, K., and Kossai, M. (2014) The oestrogen receptor alpha-regulated lncRNA NEAT1 is a critical modulator of prostate cancer. Nature Communications 5, 5383-5383

12. Choudhry, H., Albukhari, A., Morotti, M., Hider, S., Moralli, D., Smythies, J., Green, C. M., Camps, C., Buffa, F., and Ratcliffe, P. (2014) Tumor hypoxia induces nuclear paraspeckle formation through HIF-2 伪 dependent transcriptional activation of NEAT1 leading to cancer cell survival. Journal of Applied Psychology 68, 102-114

13. Sun, C., Li, S., Zhang, F., Xi, Y., Wang, L., Bi, Y., and Li, D. (2016) Long non-coding RNA NEAT1 promotes non-small cell lung cancer progression through regulation of miR-377-3p-E2F3 pathway. Oncotarget

14. Zhen, L., Yun-Hui, L., Hong-Yu, D., Jun, M., and YiLong, Y. (2016) Long noncoding RNA NEAT1 promotes glioma pathogenesis by regulating miR449b-5p/c-Met axis. Tumor Biology 37, 673-683

15. He, C., Jiang, B., Ma, J., and Li, Q. (2016) Aberrant NEAT1 expression is associated with clinical outcome in high grade glioma patients. APMIS 124

16. Jiang, P., Ping, W., Sun, X., Yuan, Z., Zhan, R., Ma, X., and Li, W. (2016) Knockdown of long noncoding RNA H19 sensitizes human glioma cells to temozolomide therapy. Oncotargets \& Therapy 9 , 
3501-3509

17. Kitange, G. J., Carlson, B. L., Schroeder, M. A., Grogan, P. T., Lamont, J. D., Decker, P. A., Wu, W., James, C. D., and Sarkaria, J. N. (2009) Induction of MGMT expression is associated with temozolomide resistance in glioblastoma xenografts. NeuroOncology 11, 281-291

18. Hegi, M. E., Janzer, R. C., Lambiv, W. L., Gorlia, T., Kouwenhoven, M. C. M., Hartmann, C., Deimling, A. V., Martinet, D., Schmutz, N. B., and Diserens, A. C. (2012) Presence of an oligodendrogliomalike component in newly diagnosed glioblastoma identifies a pathogenetically heterogeneous subgroup and lacks prognostic value: central pathology review of the EORTC_26981/NCIC_CE.3 trial. Acta Neuropathologica 123, 841-852

19. Wang, Y., and Jiang, T. (2013) Understanding high grade glioma: molecular mechanism, therapy and comprehensive management. Cancer Letters 331, 139-146

20. Stupp, R., Mason, W. P., Van, d. B., Martin J., Weller, M., Fisher, B., Taphoorn, M. J. B., Belanger, K., Brandes, A. A., Marosi, C., and Bogdahn, U. (2005) Radiotherapy plus concomitant and adjuvant temozolomide for glioblastoma. Dkgest of the World Latest Medical Information 352, 987-996

21. Strowd, R. E., Blackwood, R., Brown, M., Harmon, M., Lovato, J., Yalcinkaya, T., and Lesser, G. (2013) Impact of temozolomide on gonadal function in patients with primary malignant brain tumors. Journal of Oncology Pharmacy Practice Official Publication of the International Society of Oncology Pharmacy Practitioners 19, 321-327

22. Wang, Y., Wang, Y., Li, J., Zhang, Y., Yin, H., and Han, B. (2015) CRNDE, a long-noncoding RNA, promotes glioma cell growth and invasion through mTOR signaling. Cancer letters 367, 122-128

23. Xu, W. H., Zhang, J. B., Dang, Z., Li, X., Zhou, T., Liu, J., Wang, D. S., Song, W. J., and Dou, K. F. (2014) Long non-coding RNA URHC regulates cell proliferation and apoptosis via ZAK through the ERK/MAPK signaling pathway in hepatocellular carcinoma. International Journal of Biological Sciences 10, 664-676

24. Z, L. (2016) TUG1: a pivotal oncogenic long noncoding RNA of human cancers. Cell Proliferation

\section{$49,471-475$}

25. Cheng, Y., Jutooru, I., Chadalapaka, G., Corton, J. C., and Safe, S. (2015) The long non-coding RNA HOTTIP enhances pancreatic cancer cell proliferation, survival and migration. Oncotarget 6, 10840-10852

26. Li, Z. (2015) The long non-coding RNA HOTTIP promotes progression and gemcitabine resistance by regulating HOXA13 in pancreatic cancer. Journal of Translational Medicine 13, 1-16

27. Pan, J., Li, X., Wu, W., Xue, M., Hou, H., Zhai, W., and Chen, W. (2016) Long non-coding RNA UCA1 promotes cisplatin/gemcitabine resistance through CREB modulating miR-196a-5p in bladder cancer cells. Cancer Letters 382, 64-76

28. Zhang, X. W., Bu, P., Liu, L., Zhang, X. Z., and Li, J. (2015) Overexpression of long non-coding RNA PVT1 in gastric cancer cells promotes the development of multidrug resistance. Biochemical \& Biophysical Research Communications 462, 227232

29. Zhai, H. Y., Sui, M. H., Yu, X., Qu, Z., Hu, J. C., Sun, H. Q., Zheng, H. T., Zhou, K., and Jiang, L. X. (2016) Overexpression of Long Non-Coding RNA TUG1 Promotes Colon Cancer Progression. Medical Science Monitor International Medical Journal of Experimental \& Clinical Research 22, 3281-3287

30. Dong, S., Qu, X., Li, W., Zhong, X., Li, P., Yang, S., Chen, X., Shao, M., and Zhang, L. (2015) The long non-coding RNA, GAS5, enhances gefitinibinduced cell death in innate EGFR tyrosine kinase inhibitor-resistant lung adenocarcinoma cells with wide-type EGFR via downregulation of the IGF-1R expression. Journal of Hematology \& Oncology 8, 1-13

31. Zhang, Y., Ma, M., Liu, W., Ding, W., and Yu, H. (2014) Enhanced expression of long noncoding RNA CARLo-5 is associated with the development of gastric cancer. International Journal of Clinical \& Experimental Pathology 7, 8471-8479

32. L, P., F, P., E, R., A, D. P., S, A., C, F., F, V., S, I., R, S., and G, B. (2012) BMP2 sensitizes glioblastoma stem-like cells to Temozolomide by affecting HIF- $1 \alpha$ stability and MGMT expression. Cell Death \& Disease 3, $\mathrm{e} 412$ 